Old Dominion University

ODU Digital Commons

Physics Faculty Publications

Physics

1995

\title{
Evidence for Virtual Compton Scattering from Proton
}

J.F.J. van den Brand

R. Ent

P. L. Anthony

R. G. Arnold

J. Arrington

See next page for additional authors

Follow this and additional works at: https://digitalcommons.odu.edu/physics_fac_pubs

Part of the Astrophysics and Astronomy Commons, Elementary Particles and Fields and String Theory Commons, Nuclear Commons, and the Quantum Physics Commons

\section{Original Publication Citation}

Vandenbrand, J. F. J., Ent, R., Anthony, P. L. ... Kuhn, S., Lee, K., Zeidman, B. (1995). Evidence for virtual Compton scattering from the proton. Physical Review D, 52(9), 4868-4871. https://doi.org/10.1103/ PhysRevD.52.4868

This Article is brought to you for free and open access by the Physics at ODU Digital Commons. It has been accepted for inclusion in Physics Faculty Publications by an authorized administrator of ODU Digital Commons. For more information, please contact digitalcommons@odu.edu. 


\section{Authors}

J.F.J. van den Brand, R. Ent, P. L. Anthony, R. G. Arnold, J. Arrington, E. J. Beise, J. E. Belz, P. E. Bosted, H. J. Bulten, M. S. Chapman, K. P. Coulter, F. S. Dietrich, M. Epstein, B. W. Filippone, H. Gao, R. A. Gearhart, D. F. Geesaman, J.-O. Hansen, R. J. Holt, H. E. Jackson, C. E. Jones, C. E. Keppel, E. R. Kinney, S. Kuhn, K. Lee, W. Lorenzon, A. Lung, N.C.R. Makins, D.J. Margaziotis, R.D. McKeown, R.G. Milner, B. Mueller, J. Napolitano, J. Nelson, T. G. O'Neill, V. Pavavassiliou, G. G. Petratos, D. H. Potterveld, S. E. Rock, M. Spengos, Z. M. Szalata, L. H. Tao, K van Bibber, D. A. Wasson, J.L. White, and B. Zeidman 


\title{
Evidence for virtual Compton scattering from the proton
}

J. F. J. van den Brand, ${ }^{1}$ R. Ent, ${ }^{2, a}$ P. L. Anthony, ${ }^{3}$ R. G. Arnold, ${ }^{4}$ J. Arrington, ${ }^{5}$ E. J. Beise, ${ }^{5, b}$ J. E. Belz, ${ }^{5, c}$ P. E. Bosted ${ }^{4}$ H.-J. Bulten, ${ }^{1}$ M. S. Chapman, ${ }^{2}$ K. P. Coulter, ${ }^{6},{ }^{d}$ F. S. Dietrich, ${ }^{3}$ M. Epstein, ${ }^{7}$ B. W. Filippone, ${ }^{5}$ H. Gao, ${ }^{5, e}$ R. A. Gearhart, ${ }^{8}$ D. F. Geesaman,${ }^{6}$ J.-O. Hansen, ${ }^{2, f}$ R. J. Holt, ${ }^{6, e}$ H. E. Jackson, ${ }^{6}$ C. E. Jones, ${ }^{1, f}$ C. E. Keppel, ${ }^{4, \mathrm{~g}}$ E. R. Kinney, ${ }^{9}$ S. Kuhn, ${ }^{10, \mathrm{~h}}$ K. Lee ${ }^{2}$ W. Lorenzon, ${ }^{5, \mathrm{i}}$ A. Lung, ${ }^{4, j}$ N. C. R. Makins, ${ }^{2, f}$ D. J. Margaziotis, ${ }^{7}$ R. D. McKeown,${ }^{5}$ R. G. Milner, ${ }^{2}$ B. Mueller,${ }^{5}$ J. Napolitano, ${ }^{11}$ J. Nelson, ${ }^{2, k}$ T. G. O'Neill, ${ }^{5, f}$ V. Papavassiliou, ${ }^{6}$ G. G. Petratos, ${ }^{8,1}$ D. H. Potterveld, ${ }^{6}$ S. E. Rock, ${ }^{4}$ M. Spengos, ${ }^{4, j}$ Z. M. Szalata, ${ }^{4}$ L. H. Tao, ${ }^{4}$ K. van Bibber, ${ }^{3}$ D. A. Wasson, ${ }^{2, m}$ J. L. White, ${ }^{4}$ and B. Zeidman ${ }^{6}$

${ }^{1}$ Department of Physics, University of Wisconsin, Madison, Wisconsin 53706

${ }^{2}$ Massachusetts Institute of Technology, Cambridge, Massachusetts 02139

${ }^{3}$ Lawrence Radiation Laboratory, Livermore, California 94550

${ }^{4}$ Department of Physics, The American University, Washington, D. C. 20016

${ }^{5}$ California Institute of Technology, Pasadena, California 91125

${ }^{6}$ Physics Division, Argonne National Laboratory, Argonne, Mlinois 60439

${ }^{7}$ Department of Physics, California State University, Los Angeles, California 90032

${ }^{8}$ Stanford Linear Accelerator Center, Stanford, California 94309

${ }^{9}$ Department of Physics, University of Colorado, Boulder, Colorado 80309

${ }^{10}$ Stanford University, Stanford, California 94305

${ }^{11}$ Department of Physics, Rensselaer Polytechnic Institute, Troy, New York 12180

(Received 4 April 1995)

\begin{abstract}
In virtual Compton scattering an electron is scattered off a nucleon such that the nucleon emits a photon. We show that these events can be selected experimentally, and present the first evidence for virtual Compton scattering from the proton in data obtained at the Stanford Linear Accelerator Center. The angular and energy dependence of the data is well described by a calculation that includes the coherent sum of electron and proton radiation.
\end{abstract}

PACS number(s): 13.60.Fz, 14.20.Dh

A central question in subatomic physics is the description of the nucleon in terms of quarks and gluons. Traditionally, the electromagnetic structure of the nucleon has been investigated with elastic electron scattering, deep-

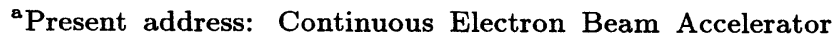
Facility, Newport News, VA 23606.

${ }^{b}$ Present address: Physics Department, University of Maryland, College Park, MD 20742.

${ }^{\mathrm{c}}$ Present address: Department of Physics, University of Colorado, Boulder, CO 80309.

${ }^{\mathrm{d}}$ Present address: Department of Physics, University of Michigan, Ann Arbor, MI 48109.

${ }^{e}$ Present address: Department of Physics, University of Illinois, Urbana, IL 61801.

${ }^{f}$ Present address: Physics Division, Argonne National Laboratory, Argonne, IL 60439.

${ }^{8}$ Present address: Physics Department, Virginia Union University, Richmond, VA 23220.

${ }^{\text {h }}$ Present address: Old Dominion University, Norfolk, VA 23529.

${ }^{i}$ Present address: Physics Department, University of Pennsylvania, Philadelphia, PA 19106.

${ }^{j}$ Present address: Caltech, Pasadena, CA 91125.

${ }^{k}$ Present address: SLAC, Stanford, CA 94309.

${ }^{1}$ Present address: Physics Department, Kent State University, Kent, OH 44242.

${ }^{m}$ Present address: Arete, Marina del Rey, CA 90292.
}

inelastic scattering, and real Compton scattering. Elastic scattering has yielded the electromagnetic form factors $[1,2]$ related to charge and current distributions in the nucleon. Deep-inelastic scattering has established the existence of pointlike partons inside the nucleon [3], and provides access to the momentum and spin distributions of these partons. Real Compton scattering has provided information on the electric and magnetic polarizabilities of the nucleon $[4,5]$ which enter as the leading corrections to the low-energy theorem (LET) [6].

Here, the virtual Compton (VC) scattering process $\gamma^{*} N \rightarrow \gamma N$ is discussed, where a photon with virtuality $Q^{2}$ is absorbed on a nucleon which subsequently radiates a real photon with energy (momentum) $\omega^{\prime}\left(\overrightarrow{q^{\prime}}\right)$. This process is intrinsically a rich probe of nucleon dynamics, since one can independently map out the dependence of the VC process on $Q^{2}$, the virtual photon's polarization, and the invariant mass of the hadronic intermediate state $W$, given by

$$
W^{2}=M^{2}+2 \omega^{\prime}\left(E_{p}-p_{z}^{f}\right)
$$

Here $p_{z}^{f}, E_{p}$, and $M$ are respectively the momentum component along the $\overrightarrow{q^{\prime}}$ direction, the energy, and the mass of the outgoing proton. In addition, one can experimentally select the polarization of incident beam, target, or recoiling proton. In the limit $Q^{2} \rightarrow 0$ the $\mathrm{VC}$ reduces to the real Compton process, while in the limit $W^{2} \rightarrow M^{2}$ it becomes equivalent to elastic electron-proton scattering.

The VC process provides a particularly interesting 
probe of nucleon structure, since it represents a fully electromagnetic exclusive reaction, where significant initialand final-state interactions are absent [7]. Already more than 40 years ago Drell recognized the sensitivity of this reaction to negative energy states [8]. After this, several authors have discussed the virtue of this reaction to study various aspects of nucleon structure. In the nonperturbative QCD (NPQCD) regime, below the $\pi^{0}$-production threshold, the VC process allows the determination of electromagnetic polarizabilities through the measurement of $q^{\prime}$-dependent contributions to the LET [6,9]. These observables are determined by the virtual excitation spectrum of the nucleon. In the region above the $\pi^{0}$-production threshold VC scattering allows the study of $s$-channel effects of baryon resonances [10]. Here $W^{2}$ can be chosen to optimize the sensitivity to the electromagnetic decay of a particular resonance (e.g., $W^{2} \approx M_{\Delta}^{2}$ ). In the hard-scattering region, $Q^{2}>1(\mathrm{GeV} / c)^{2}, \mathrm{VC}$ scattering can be employed to investigate the perturbative QCD (PQCD) description of the nucleon wave function [11]. However, up to now no data have been obtained for VC scattering. Recently, a discussion of this process to test nucleon models in the NPQCD [12] and PQCD region $[13,14]$ has started, in large part motivated by the advent of high-intensity continuous beam electron accelerators [e.g., the Continuous Electron Beam Accelerator Facility (CEBAF)].

The reaction $e N \rightarrow e N \gamma$ is dominated by contributions from the photons radiated by the incoming and outgoing electrons. The corresponding lowest-order Feynman diagrams can be calculated exactly using QED and are termed Bethe-Heitler contributions. The VC contributions are due to photon radiation by the nucleons and can only be unambiguously calculated in the soft-photon limit, where the cross section is independent of nucleonstructure ambiguities. In general they involve a sum over all possible intermediate hadronic states. The calculation of the VC contribution requires either solving QCD or a realistic nucleon model to describe its dynamical behavior and must also take into account the propagation of intermediate nucleon resonances and other intermediate states. Note that the reaction $e N \rightarrow e N \gamma$ will also provide phase information on the nucleon matrix elements, due to the interference of the (calculable) Bethe-Heitler and VC contributions. This phase information can be used to determine the real part of the Compton amplitude [15].

Experimentally, the reaction $e N \rightarrow e N \gamma$ can be kinematically determined by detecting a scattered electron in coincidence with a proton using high-resolution magnetic spectrometers. The NE-18 experiment at the Stanford Linear Accelerator Center (SLAC) measured the $A\left(e, e^{\prime} p\right)$ reaction for several target nuclei, using the $\mathbf{1 . 6}$ $\mathrm{GeV} / c$ and $8 \mathrm{GeV} / c$ spectrometers [16] in coincidence. Data for the ${ }^{1} H\left(e, e^{\prime} p\right)$ reaction were taken in order to calibrate the coincidence detection setup, for an incident (scattered) electron energy of $2.015(1.400) \mathrm{GeV}$, scattering angle $\theta_{e}=37.29^{\circ}$ and $Q^{2}=1.15(\mathrm{GeV} / c)^{2}$. A $2 \mu \mathrm{A}$ electron beam was incident on a $4 \mathrm{~cm} \mathrm{LH} \mathrm{LH}_{2}$ target and a total of $5 \times 10^{4}$ counts were measured. The electron beam had a duty factor of $2 \times 10^{-4}$, and an energy resolution of $\pm 0.1 \%$. A coincidence time resolution of $0.8 \mathrm{~ns}$ [full width at half maxium (FWHM)] was achieved. Uncertainties (FWHM) are $0.1 \%$ for the beam energy, and 0.5 mrad for the scattering and outgoing proton angle. Further details on the detector setup and the data analysis can be found in Refs. [17-19]. The measurement of the VC contribution is complicated due to the fact that an abundance of photons will be radiated by the incoming and scattered electrons. This will lead to radiation tails in the missing-energy and momentum plane, defined by $E_{m}=\omega-E_{p^{\prime}}+M$ and $\overrightarrow{p_{m}}=\vec{q}-\overrightarrow{p^{\prime}}$, with $\omega(\vec{q})$ the energy (momentum) transfer and $E_{p^{\prime}}\left(\overrightarrow{p^{\prime}}\right)$ the final proton

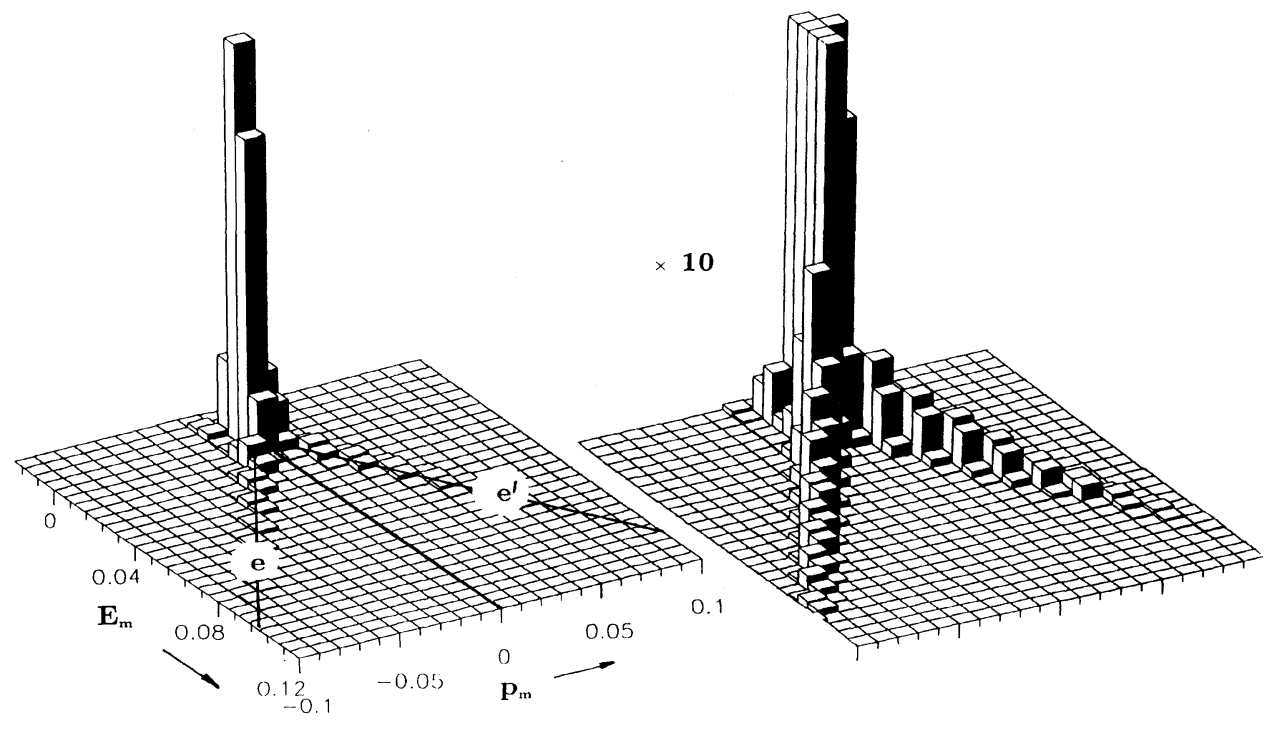

FIG. 1. Event distribution versus $E_{m}$ and $p_{m}$ (in $\mathrm{GeV}$ ) without radiative corrections for the reaction ${ }^{1} \mathrm{H}\left(e, e^{\prime} p\right)$. The two radiative tails due to photon emission of the electron before and after the interaction can be observed. The sign of $\overrightarrow{p_{m}}$ is $+(-)$ in case the angle of $\overrightarrow{p_{m}}$, with respect to the incident electron, is $<(>) \theta_{e} / 2$. 
energy (momentum). For the reaction $e N \rightarrow e N \gamma$ one has $\omega^{\prime}=E_{m}$ and $\overrightarrow{q^{\prime}}=\overrightarrow{p_{m}}$. Figure 1 demonstrates this effect with the data recently obtained at SLAC. The tails due to radiation of the incident and scattered electrons are readily observed. It is seen that the radiated events are distributed along lines with $E_{m}=\left|p_{m}\right|$ as required for real photons.

The angular distribution of the emitted photons can be reconstructed from the measured $\overrightarrow{p_{m}}$. We will only consider events with a missing energy $\left(\simeq \omega^{\prime}\right)$ larger than $20 \mathrm{MeV}$ since in the region $\omega^{\prime} \rightarrow 0 \mathrm{MeV}$ the experimental resolutions, $8 \mathrm{MeV}(10 \mathrm{MeV} / c)$ in missing energy (momentum), do not permit an accurate reconstruction of the photon angle. Furthermore, applying a lower cutoff in $\omega^{\prime}$ is needed to minimize effects due to multiphoton emission. Figure 2 shows the angular distribution of the count rate for events with $E_{m}>30 \mathrm{MeV}$. The invariant mass of the photons has been reconstructed from the kinematics and the photon events are well separated from the $\pi^{0}$-production channel. It is seen that electron radiation is predominantly in the direction of the initial and final electrons, in accordance with the peaking approximation [20]. Note that a broad distribution of events is seen in the direction of the outgoing proton.

Next, the angular distributions were calculated in the soft-photon limit. Here, the proton contribution corresponds to radiation from a Dirac particle with the usual form factors $F_{1}\left(Q^{2}\right)$ and $F_{2}\left(Q^{2}\right)$. The standard formalism by Mo and Tsai [21] was extended to coincidence $\left(e, e^{\prime} p\right)$ reactions [22], enabling a calculation of the softphoton differential cross section of the ${ }^{1} \mathrm{H}\left(e, e^{\prime} p\right) \gamma$ reaction. This differential cross section was reduced to the

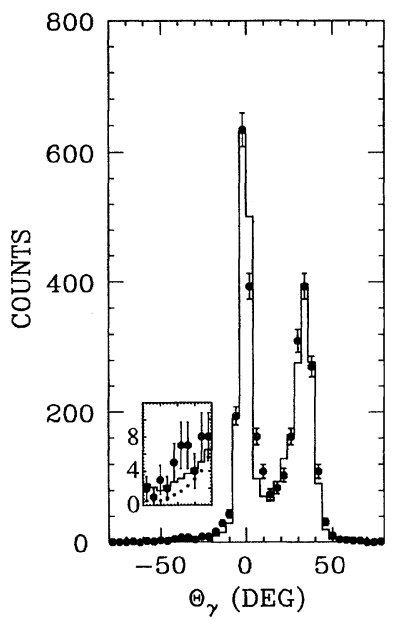

FIG. 2. Calculated angular distribution of radiated events in comparison with NE-18 data for $\omega^{\prime}>30 \mathrm{MeV}$. The solid (dotted; see inset) curve shows the prediction in the soft-photon limit for Bethe-Heitler and VC contributions (Bethe-Heitler only). The central angle $\theta_{\gamma}$ for the incident (scattered) electron and outgoing proton are $0^{\circ}\left(37.29^{\circ}\right)$ and $-43.29^{\circ}$, respectively. Note that the inset shows the region $-60^{\circ}<\theta_{\gamma}<-20^{\circ}$ with a different vertical scale. cross section for (multi)photon emission with total energy $\omega^{\prime}$ and angle $\theta_{\gamma}$ in the scattering plane. For this we used polar coordinates, integrating over the range of $\tan \left(\phi_{\gamma}\right)$ accepted by the phase space. Effects arising from imperfect knowledge of the phase space were suppressed through an energy cut, $\omega^{\prime}<80 \mathrm{MeV}$, applied for radiated photons along the incident electron beam. The absolute photon rate was calculated by folding the cross section over the spectrometer acceptances and including experimental resolutions. No normalization factors were used. The data has a systematic uncertainty of $6.6 \%$, which is mainly due to uncertainty in the detection phase space. As Fig. 2 shows, the agreement between data and simulation is excellent.

Having verified that we satisfactorily understand the angular distribution of radiated events (note that only about $3 \times 10^{3}$ out of a total of $5 \times 10^{4}$ events have radiated more than $30 \mathrm{MeV}$ ), a region sensitive to the $\mathrm{VC}$ scattering process is selected. That such a region exists is illustrated in Fig. 3, which shows the calculated angular distribution of radiated photons in the single-photon limit. For this, the total radiative effects can be divided into three parts, one solely due to electron radiation $\left(\delta_{e e}\right)$, one due to the contribution from electron-proton interference $\left(\delta_{e p}\right)$, and one due to the contribution from direct proton radiation $\left(\delta_{p p}\right)$ only. It is seen that radiation from the proton is dominant for photon angles less than $-80^{\circ}$, while in the photon angle range between $-60^{\circ}$ and $-20^{\circ}$ one expects about a similar amount of photons originating from VC processes as from Bethe-Heitler processes (see also the inset of Fig. 2). We would like to point out the differences between Figs. 2 and 3 . In Fig. 3 the prominent dip along the proton angle reflects the character of dipole radiation boosted along the particle's momentum, emphasized in the single-photon limit. The electron radiation peaks $\left(\delta_{e e}\right)$ also have sharp minima at their maxima, but because the boost of the dipole pattern is so large, the minima are so narrow that they are not shown in the figure. In Fig. 2 a complete angular distribution of radiated photons is calculated, where all multiphoton contributions are taken into account.

In Fig. 4 we show the number of radiated events versus

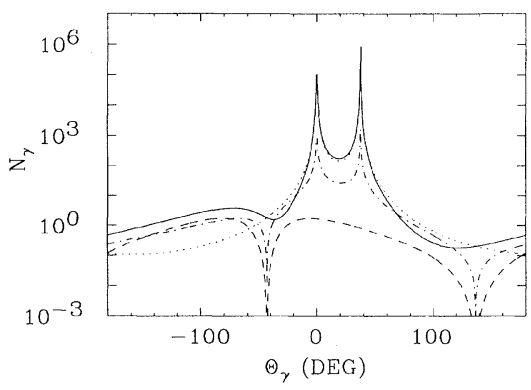

FIG. 3. Angular distribution of radiated photons in the single-photon limit. No acceptance effects are taken into account. The various curves denote the $\delta_{e e}$ (dotted), $\delta_{e p}$ (dot-dashed), and $\delta_{p p}$ (dashed) contributions. The solid curve shows the coherent sum of the various contributions. 


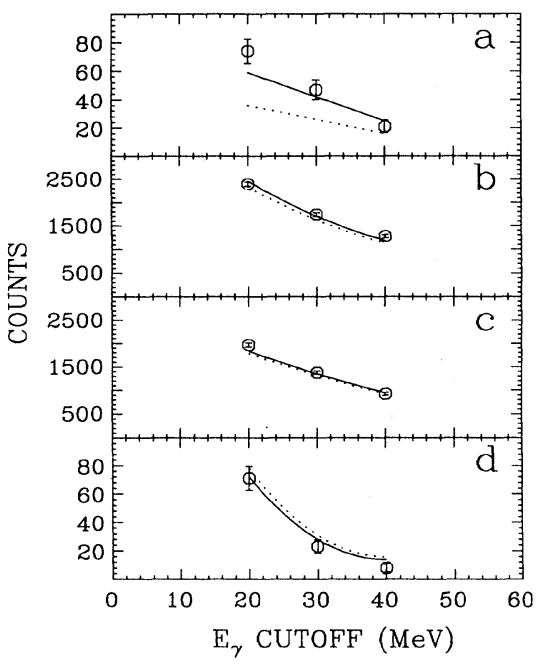

FIG. 4. Number of radiated events versus the cutoff energy $\omega^{\prime}$, in the intervals $-60^{\circ}<\theta_{\gamma}<-20^{\circ}$ [radiation along the proton direction (a)], $-20^{\circ}<\theta_{\gamma}<20^{\circ}$ [radiation along the incident electron direction (b)], $20^{\circ}<\theta_{\gamma}<50^{\circ}$ [radiation along the scattered electron direction (c)], and the region $50^{\circ}<\theta_{\gamma}<90^{\circ}(\mathrm{d})$. The solid (dotted) curve shows the prediction in the soft-photon limit for Bethe-Heitler and VC contributions (Bethe-Heitler only).

the cutoff energy $\omega^{\prime}$, in various intervals $\Delta \theta_{\gamma}$. The dotted curve shows the prediction when only Bethe-Heitler contributions are taken into account. This calculation gives a good description of the energy dependence of the data measured in the intervals $-20^{\circ}<\theta_{\gamma}<20^{\circ}$ [Fig. 4(b)], and $20^{\circ}<\theta_{\gamma}<50^{\circ}$ [Fig. 4(c)], corresponding to radiation along the incident and scattered electron. A good description is also obtained for the radiated photons in the angular range $50^{\circ}<\theta_{\gamma}<90^{\circ}$ [Fig. 4(d)], which is

[1] R.G. Arnold et al., Phys. Rev. Lett. 57, 174 (1986).

[2] P.E. Bosted et al., Phys. Rev. Lett. 68, 3841 (1992); A. Lung et al., ibid. 70, 718 (1993).

[3] J.I. Friedman, H.W. Kendall, and R.E. Taylor, Rev. Mod. Phys. 63, 573 (1990).

[4] A. Klein, Phys. Rev. 99, 998 (1955); V.A. Petrun'kin, Sov. J. Part. Nucl. 12, 278 (1981).

[5] F.J. Federspiel et al., Phys. Rev. Lett. 67, 1511 (1991); A. Zieger et al., Phys. Lett. B 278, 34 (1992).

[6] F.E. Low, Phys. Rev. 96, 1428 (1954); M. Gell-Mann and M.L. Goldberg, ibid. 96, 1433 (1954).

[7] S.J. Brodsky in, Proceedings of the Workshop on 'CEBAF at Higher Energies', edited by N. Isgur and P. Stoler (CEBAF, Newport News, Virginia, 1994), p. 153.

[8] S.D. Drell, Phys. Rev. 87, 753 (1952).

[9] P.A.M. Guichon (private communication); and (unpublished).

[10] S.J. Brodsky, A.C. Hearn, and R.G. Parsons, Phys. Rev. 187, 1899 (1969); S. Capstick and B.D. Keister, Phys. Rev. D 46, 84 (1992).

[11] A.N. Kronfeld and B. Nizic, Phys. Rev. D 44, 3445 (1991). especially sensitive to the effects of experimental resolutions. However, the calculation with Bethe-Heitler contributions only shows a deficiency in the description for radiation in the angular range $-60^{\circ}<\theta_{\gamma}<-20^{\circ}$ [Fig. 4(a)], which corresponds to radiation along the proton direction. The solid curves represent the prediction in the soft-photon limit for both Bethe-Heitler and VC contributions. The good agreement between data and prediction of the number of counts when proton radiation is included clearly demonstrates the experimental viability of the technique even with the $2 \times 10^{-4}$ duty factor available at the SLAC end station. The number of counts in this regime is too low to allow for a meaningful study of the nucleon structure. However, with the advent of the high-luminosity beam at CEBAF and the projected highresolution spectrometers, high statistics virtual Compton scattering experiments should be possible $[12,14]$.

In summary, we have presented the first data on the virtual Compton scattering process from the proton. We have shown that these experiments are possible, and that one can select a region where the virtual Compton contributions dominate. The results give important impetus to future measurements of the virtual Compton scattering cross section in the reaction $e N \rightarrow e N \gamma$ which will allow for a clean test of our understanding of the nucleon.

This work was supported in part by the Department of Energy under Contracts No. W-31-109-ENG38 (Argonne), No. DE-FG02-86ER40269 (Colorado), No. W-2705-Eng-48 (LLNL), No. DE-AC02-76ER03069 (MIT), No. DE-AC03-76SF00515 (SLAC), No. DEFG03-88ER40439 (Stanford) aid by the National Science Foundation under Grants No. PHY-9014406 and No. PHY-9114958 (American), No. PHY-9115574 (Caltech), No. PHY-9101404 (CSLA), No. PHY-9208119 (RPI), No. PHY-9316221 (Wisconsin). R.G.M. acknowledges the support from the NSF Presidential Young Investigator program. B.W.F. acknowledges the support of the Sloan Foundation.
[12] CEBAF experiment 94-011, J.F.J. van den Brand, H.J. Bulten, and R. Ent, spokespersons.

[13] H. Fonvieille et al., in The ELFE Project: An Electron Laboratory for Europe: Physics with a 15-30 GeV High Intensity Continuous Beam Electron Accelerator, Proceedings, edited by J. Arvieux and E. De Sanctis, Italian Physical Society Conference Society Vol. 44 (Italian Physical Society, Rome, 1993), p. 137.

[14] CEBAF experiment 93-050, P.Y. Bertin, G. Fournier, and C.E. Hyde-Wright, spokespersons.

[15] S.J. Brodsky, F.E. Close, and J.F. Gunion, Phys. Rev. D 5, 1385 (1972).

[16] NPAS Users Guide, SLAC Report No. 269, 1984 (unpublished).

[17] N.C.R. Makins et al., Phys. Rev. Lett. 72, 1986 (1994).

[18] N.C.R. Makins, Ph.D. thesis, Massachusetts Institute of Technology, 1994.

[19] T.G. O'Neill, Ph.D. thesis, California Institute of Technology, 1994.

[20] L.I. Schiff, Phys. Rev. 87, 750 (1952).

[21] L.W. Mo and Y-S. Tsai, Rev. Mod. Phys. 41, 205 (1969).

[22] D.A. Wasson et al. (unpublished). 